\title{
Blockade of Autoantibody-Initiated Tissue Damage by Using Recombinant Fab Antibody Fragments against Pathogenic Autoantigen
}

\author{
Gang Wang, ${ }^{\star \dagger}$ Hideyuki Ujiie, ${ }^{*}$ Akihiko Shibaki, ${ }^{\text {* }}$ \\ Wataru Nishie, ${ }^{*}$ Yasuki Tateishi, ${ }^{*}$ \\ Kazuhiro Kikuchi, ${ }^{*}$ Qiang Li, ${ }^{*}$ James R. McMillan, ${ }^{* \dagger}$ \\ Hiroshi Morioka, ${ }^{\ddagger}$ Daisuke Sawamura, ${ }^{*}$ \\ Hideki Nakamura, ${ }^{*}$ and Hiroshi Shimizu* \\ From the Department of Dermatology, Hokkaido University \\ Graduate School of Medicine, the Creative Research Initiative \\ Sousei, ${ }^{\dagger}$ and the Faculty of Pharmaceutical Sciences, ${ }^{\ddagger}$ Hokkaido \\ University, Sapporo, Japan
}

\begin{abstract}
Activation of the complement cascade via the classical pathway is required for the development of tissue injury in many autoantibody-mediated diseases. It therefore makes sense to block the pathological action of autoantibodies by preventing complement activation through inhibition of autoantibody binding to the corresponding pathogenic autoantigen using targeted Fab antibody fragments. To achieve this, we use bullous pemphigoid (BP) as an example of a typical autoimmune disease. Recombinant Fabs against the non-collagenous 16th-A domain of type XVII collagen, the main pathogenic epitope for autoantibodies in BP, were generated from antibody repertoires of BP patients by phage display. Two Fabs, Fab-B4 and Fab-19, showed marked ability to inhibit the binding of BP autoantibodies and subsequent complement activation in vitro. In the in vivo experiments using type XVII collagen humanized BP model mice, these Fabs protected mice against BP autoantibody-induced blistering disease. Thus, the blocking of pathogenic epitopes using engineered Fabs appears to demonstrate efficacy and may lead to disease-specific treatments for antibody-mediated autoimmune diseases. (Am J Pathol 2010, 176:914-925; DOI: 10.2353/ajpath.2010.090744)
\end{abstract}

Autoimmune diseases are a major cause of morbidity and mortality in humans, affecting approximately $5 \%$ of the general population. ${ }^{1}$ In recent years, significant ad- vances have been made in our understanding of autoimmune disease pathomechanisms, especially the roles of autoantibodies, complement system, and autoresponsive T cells. For many autoimmune diseases such as systemic lupus erythematosus, rheumatoid arthritis, anti-phospholipid syndrome (APS), and bullous pemphigoid (BP), complement activation is increasingly recognized as critical to tissue injury. ${ }^{2-6}$ Studies of APS and BP, for example, showed that the classical pathway of complement activation is required for the development of tissue injury, although alternative pathways may also be involved. ${ }^{4,7-9}$

$\mathrm{BP}$ is the most common autoimmune blistering skin disease. Autoantibodies against collagen XVII (COL17) bind to dermal-epidermal junction (DEJ) components and activate the complement system that mediates a series of inflammatory events including dermal mast cell degranulation and generation of eosinophil-rich infiltrates, resulting in skin blister formation. ${ }^{10-12}$ APS is a condition characterized by recurrent miscarriage and thrombosis formation in the presence of anti-phospholipid autoantibodies, and a therapy has been proven effective to prevent the fetal loss by using heparin to inhibit anti-phospholipid antibody-induced complement

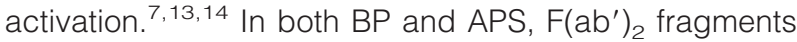
from the pathogenic autoantibodies, which lack the Fc portion necessary to activate the complement pathway, fail to initiate the disease. ${ }^{4,7}$ This suggests that preventing complement activation by blocking the binding of autoantibodies to the corresponding antigens can be a viable novel therapeutic strategy for treating these diseases.

Supported by a grant-in-aid from the Program for Promotion of Fundamental Studies in Health Sciences of the National Institute of Biomedical Innovation (NIBIO; to H.S.).

G.W. and H.U. contributed equally to this work. A.S. and H.S. contributed equally to the direction of this study.

Accepted for publication October 7, 2009.

Supplemental material for this article can be found on http://ajp. amjpathol.org

Address reprint requests to Dr. Akihiko Shibaki or Dr. Hiroshi Shimizu, Department of Dermatology, Hokkaido University Graduate School of Medicine, N15 W7, Sapporo, 060-8638 Japan. E-mail: ashibaki@med hokudai.ac.jp or shimizu@med.hokudai.ac.jp. 
Table 1. PCR Primers for the Amplification of Human Antibody Gene Repertoires

$\begin{array}{ll}\text { Primers for } \kappa & \\ \text { HK5 } & \\ \text { HK3 } & 5^{\prime} \text {-GAMATYGAGCTCACSCAGTCTCCA-3' (Sac I) } \\ \text { Primers for } \lambda & 5^{\prime} \text {-GCGCCGTCTAGAACTAACACTCTCCCCTGTTGAAGCTCTTTGTGACGGGCAAG-3' (Xba I) } \\ \text { HL5 } & \text { 5'-CASTYTGAGCTCACKCARCCGCCCTC-3' (Sac I) } \\ \text { HL3 } & \text { 5'-GAGGGATCTAGAATTATGAACATTCTGTAGG-3' (Xba I) } \\ \text { Primers for Fd } & \\ \text { H135 } & \text { 5'-CAGGTGCAGCTGGTGSAGTCTGG-3' } \\ \text { H2 } & \text { 5'-CAGGTCAACTTGAAGGATCTGG-3' } \\ \text { H4 } & \text { 5'-CAGGTGCAGCTGCAGGAGTCGGG-3' } \\ \text { VH5 } & \text { 5'-CAGGTGCAGCTCGAGSAGTCTGG-3' (Xho I) } \\ \text { HG3 } & \text { 5'-GCATGTACTAGTTTTGTCACAAGA-3' (Spe I) }\end{array}$

To allow for sequence variability, representative choices of wobble nucleotides were included in the primers $(M=A / C, K=G / T, R=A / G, S=C / G, Y=$ $\mathrm{C} / \mathrm{T}$ ). Fd fragments of human IgG were amplified in a two-step procedure. First, antisense primers $\mathrm{H} 135$, H2, and H4 were combined with HG3 for the amplification of heavy chain genes from human VH1-VH5 families and the Spe I site was introduced. In the second step, antisense primer VH5 was combined with HG3 to reamplify the heavy chain genes and introduce the Xho I site. Underlined sequences are restriction sites for the enzymes indicated in parenthesis.

The purpose of this study is to provide a proof of concept for this new strategy of treating antibody-mediated autoimmune disorders by using recombinant Fabs to block complement activation induced by pathogenic autoantibodies. Toward this end, we use BP as an example of a typical autoimmune disease. Our group has recently established a BP mouse model using a newly constructed COL17 humanized mouse. ${ }^{3}$ Here we report our success in developing Fabs against the noncollagenous 16th-A domain (NC16A) of COL17, the main pathogenic epitope of BP autoantibodies, ${ }^{15}$ for the blockade of autoantibody-initiated BP disease.

\section{Materials and Methods}

\section{Construction of Phage Antibody Libraries}

We constructed two individual Fab phage libraries from mononuclear cells isolated from two patients with active $\mathrm{BP}$. The diagnosis of BP was made by the typical clinical and histological manifestations as well as by laboratory data including anti-COL17 ELISA and indirect immunofluorescence (IIF). Phagemid expression vector P3MH, a gift from Dr. Yan Wang (Central Lab of Navy General Hospital, Beijing, China), was derived from pCOMB3H (Scripps Research Institute, La Jolla, CA) by adding 9E10/c-myc epitope for detection and a hexahistidine tag for column purification at the $3^{\prime}$ end of $\mathrm{Fd}^{16}{ }^{16}$ Using previously described methods and a set of PCR primers (Table 1), ${ }^{17-19}$ antibody genes were amplified by RT-PCR from approximately $1 \times 10^{8}$ mononuclear cells isolated from $50 \mathrm{ml}$ of peripheral blood from each patient. The phage antibody libraries were constructed by randomly combining the genes coding Fd fragments of IgG heavy chains with IgG light chain genes of either lambda or kappa DNA in equal amounts (see Supplemental Figure S1 at http://ajp.amjpathol.org.). The phagemid libraries were electroporated into E. coli XL1-Blue strain (Stratagene, La Jolla, CA), and the phage display of the libraries was performed as described elsewhere. ${ }^{17,20}$ Before amplification, the resulting libraries were examined for the coexpression of heavy and light chains by enzyme digestion and for the diversity by fingerprinting of antibody genes ( $\mathrm{Fd}$ and light chain) of 24 randomly selected single colo- nies. ${ }^{20,21}$ The amplified recombinant phages were purified from culture supernatants by polyethylene glycol precipitation and resuspended in PBS, pH 7.4, containing 1\% bovine serum albumin (BSA) and 10\% glycerol.

\section{Isolation of Phage Antibodies against NC16A Domain of Human COL17}

Recombinant fusion peptide of the human COL17 NC16A domain (rhNC16A) with glutathione S-transferase (GST) was synthesized as reported previously. ${ }^{3}$ Library panning was performed routinely. ${ }^{20-22}$ Briefly, a freshly amplified phage library (approximately $1 \times 10^{12}$ phages) was incubated for 2 hours at $37^{\circ} \mathrm{C}$ in immuno-tubes (Nunc, Roskilde, Denmark) coated with $50 \mu \mathrm{g} / \mathrm{ml}$ rhNC16A in $50 \mathrm{mmol} / \mathrm{L} \mathrm{NaHCO}_{3} \mathrm{pH}$ 9.6. After washing of the tube with $0.05 \%(\mathrm{v} / \mathrm{v})$ Tween-20 in PBS, adherent phages were eluted with $0.1 \mathrm{~mol} / \mathrm{L}$ triethylamine (SigmaAldrich, Inc., St. Louis, MO). After neutralization with 1 $\mathrm{mol} / \mathrm{L}$ Tris, $\mathrm{pH}$ 7.4, eluted phages were used to infect a fresh culture of XL1-Blue E. coli, which was amplified overnight as previously described. ${ }^{20}$ Phages were harvested from culture supernatants and then repanned against rhNC16A for three subsequent rounds as described for the original library. Individual single ampicillinresistant colonies resulting from infection of $E$. coli XL1-Blue with the eluted phage from the fourth panning round were isolated, and the binding to rhNC16A was confirmed by ELISA using HRP-conjugated anti-M13 mAb (Amersham Biosciences UK Ltd., Little Chalfont, Buckinghamshire, UK) as the developing reagent. The specific binders were screened by gene fingerprinting and sequencing to identify different clones. The variable region sequences of the separate selected clones were analyzed for homology to known human $\mathrm{V}, \mathrm{D}$, and $\mathrm{J}$ genes using the $\mathrm{V}$ BASE database (http://vbase.mrc-cpe.cam.ac.uk/).

\section{Production, Purification, and Characterization of Soluble Fab}

\section{Fab Production and Purification}

Plasmid DNA of the distinct selected clones was prepared, digested by Nhel (New England BioLabs, 
Ipswich, MA) to remove the gene III fragment, self ligated, and transformed into E. coli XL1-Blue. Clones were grown in LB containing $100 \mu \mathrm{g} / \mathrm{ml}$ ampicillin, and Fab expression was induced using $1 \mathrm{mmol} / \mathrm{L}$ isopropyl $\beta$-Dthiogalactopyranoside (IPTG, Sigma) in culture grown at $30^{\circ} \mathrm{C}$ overnight. Cells were pelleted by centrifugation, and the supernatant containing soluble Fab was taken for analysis.

Large-scale production of Fabs was achieved by growing Fab-express clones in E. coli XL1-Blue in $1 \mathrm{~L}$ of LB (plus $100 \mu \mathrm{g} / \mathrm{ml}$ ampicillin). Protein production was then induced with $1 \mathrm{mmol} / \mathrm{L}$ IPTG by culturing overnight at $30^{\circ} \mathrm{C}$ at $240 \mathrm{rpm}$. The culture supernatant was harvested by centrifugation. Fab purification was performed using HisTrap FF crude column (GE Healthcare Bio-Sciences AB, Uppsala, Sweden) according to the manufacturer's instructions. The purified Fabs were dialyzed against PBS and concentrated by Amicon ultrafiltration (Millipore, Lexington, MA) and were then characterized by SDS-PAGE and Western blotting. For animal experiments, the concentration of endotoxin in the purified Fabs was detected with the limulus amoebocyte lysate test using the QCL-1000 kit (Cambrex Bio Science Walkersville, Inc., Walkersville, MD), and endotoxin removal was performed by using Detoxi-Gel AffinityPak column (Pierce, Rockford, IL), where necessary.

\section{ELISA}

The binding activity and specificity of Fabs was confirmed by ELISA assay. ELISA plate wells were coated with $5 \mu \mathrm{g} / \mathrm{ml}$ rhNC16A in $50 \mathrm{mmol} / \mathrm{L} \mathrm{NaHCO}_{3} \mathrm{pH}$ 9.6. Recombinant mouse NC16A (rmNC16A), GST, and BSA were used as negative control antigens at similar concentrations. Supernatant containing Fabs or appropriately diluted purified Fabs was incubated on ELISA plates. After washing, plates were developed with HRPconjugated mAbs to human lambda light chain (Kirkegaard \& Perry Laboratories, Gaithersburg, MD) or kappa light chain (Bethyl Laboratories, Inc., Montgomery, TX) and o-phenylenediamine substrate (Wako, Osaka, Japan). Absorbance was read at $492 \mathrm{~nm}$.

\section{Western Blotting}

Western blotting was performed as previously described. Briefly, recombinant proteins were electrophoresed on SDS-PAGE and electrotransferred onto nitrocellulose membrane. The blots were blocked with $5 \%$ milk in TBS/T and incubated for 1 hour with the diluted Fabs at room temperature. After washing, the blots were incubated with HRP-conjugated mAbs to human lambda light chain or kappa light chain. The bound antibodies were detected by the Phototope Western Detection Systems (Cell Signaling Technology, Inc., Danvers, MA).

\section{Epitope Mapping}

Epitope mapping studies were performed using the standard Western blotting protocol described above. The
NC16A domain of human COL17 was divided into subregions as described by Giudice et al. ${ }^{15,23}$ The expression vectors NC16A1, NC16A2, NC16A2.5, and NC16A3, which respectively correspond to amino acid segments 490 to 506,507 to 520,514 to 532 , and 521 to 534, were gifts from Dr. George J Giudice (Medical College of Wisconsin, Milwaukee). Affinity purified products of recombinant human NC16A and its subregions were electrophoresed and electrotransferred to nitrocellulose membrane. The membranes were then probed with Fabs and allowed to react with HRP-conjugated secondary mAbs to human lambda light chain or kappa light chain.

\section{Immunogold Electron Microscopy}

Normal human skin samples were processed for postembedding immunoelectron microscopy as previously described. ${ }^{24,25}$ Briefly, cryofixed cryosubstituted samples were embedded in Lowicryl K11M resin and polymerized at $-60^{\circ} \mathrm{C}$ under UV light. Selected blocks were used to produce ultrathin sections that were incubated with Fabs $(80 \mu \mathrm{g} / \mathrm{ml})$, diluted in PBS-based buffer, and washed four times (five minutes each). Further incubations were performed using rabbit anti-c-myc tag antibody (Santa Cruz Biotechnology, Santa Cruz, CA) followed by four washes and further incubation with $5-\mathrm{nm}$ gold-conjugated antibody for immunogold labeling (Biocell, Cardiff, UK) diluted 1 in 200 in TBS buffer. Other primary anti-COL17 antibodies included for comparison were HD4 233, 1D1, and 1A8C, each of which recognizes different domains of human COL17 (extracellular domain close to the C-terminal, mid portion, and cytoplasmic domains, respectively). ${ }^{26}$ Sections were viewed under a Hitachi H-7100 transmission electron microscope (Hitachi, Tokyo, Japan) at $80 \mathrm{KV}$.

\section{Immunofluorescence}

Five- $\mu \mathrm{m}$ cryosections of OCT-embedded skin were cut and placed onto microscope slides and subjected to IF studies. IIF using Fabs was performed on the skin samples from human or COL17 humanized mice using a standard protocol. FITC conjugated secondary antibodies against human lambda light chain (DakoCytomation, Glostrup, Denmark), kappa light chain (Invitrogen Corp., Carlsbad, CA), or c-myc tag (Santa Cruz Biotechnology) were used as detection reagents.

\section{Surface Plasmon Resonance Analysis}

Affinity of the generated Fabs was determined by BIAcore assay. The on and off rate constants $\left(k_{\text {on }}\right.$ and $\left.k_{\text {off }}\right)$ for binding of the Fabs to rhNC16A were determined by a BIAcore 2000 instrument (Biacore AB, Uppsala, Sweden). For analysis of the interaction kinetics, Fabs in various concentrations $(100,80,60,50$, and $40 \mathrm{nmol} / \mathrm{L}$ ) were injected over the immobilized antigen at a flow rate of $20 \mu \mathrm{l} / \mathrm{min}$ using HBS-EP buffer (Biacore AB). The association and dissociation phase data were fitted simultaneously to a 1:1 Langmuir global model by using the 
BIAevaluation software. The affinities (dissociation constant, $K_{\mathrm{D}}$ ) were calculated from the ratio of the rate constants of association and disassociation $\left(k_{\mathrm{on}} / k_{\mathrm{off}}\right)$.

\section{Functional Analysis of Fabs in Vitro}

\section{Preparation of BP Autoantibodies}

BP autoantibodies were purified from either pooled sera from 20 patients or were included as separate serum samples from three patients with active BP. Briefly, the total IgG fraction from BP sera was prepared by affinity chromatography using HiTrap Protein G HP column (Amersham Biosciences UK Limited). Then, BP autantibodies against the COL17 NC16A peptide were affinity purified from the IgG fraction using HiTrap NHS-activated HP column (Amersham Biosciences UK Ltd.) precoated with rhNC16A according to the manufacturer's instructions. ${ }^{3}$ The NC16A affinity purified BP autoantibodies were dialyzed against PBS and concentrated by Amicon ultrafiltration (Millipore). These NC16A affinity purified BP autoantibodies were designated as BP antibodies (BPAbs), and used for in vitro inhibition experiments.

For the in vivo experiments using whole BP-lgG fractions as the pathogenic autoantibodies, serum samples were collected from another $10 \mathrm{BP}$ patients and total IgG fraction was prepared using HiTrap NHS-activated HP column. This was designated as BP-IgG. Binding activity with different autoantigens was tested by ELISA and Western blotting. The BP-IgG from all ten of the serum samples bound to human COL 17, and the BP-lgG from seven of the ten serum samples also reacted with BP230. The binding of the BP-lgG with the subdomains of NC16A (NC16A-1, -2, -2.5, -3, as described by Giudice et al ${ }^{15}$ ) was further studied. All ten of the serum samples bound to NC16A-2 and NC16A-2.5. In addition, two of the ten serum samples also bound to NC16A-1 or -3 . When the pooled IgG from these ten patients was first incubated with the NC16A domain of COL17 overnight at $4^{\circ} \mathrm{C}$, the reaction with the NC16A domain was markedly reduced, whereas binding to the full length COL17 was unchanged by Western blotting. This indicates that the BP-IgG recognize numerous epitopes on both COL17 and BP230 antigens, and that there exist autoantibodies recognizing different epitopes both within and outside of the COL17 NC16A domain.

\section{Inhibition ELISA}

To check the competition effect of Fabs on the binding of BPAbs to rhNC16A, an inhibition ELISA was performed by incubating purified BPAbs $(8 \mu \mathrm{g} / \mathrm{ml})$ with 0 to $32 \mu \mathrm{g} / \mathrm{ml}$ Fabs on rhNC16A ELISA plates. To detect the IgG autoantibodies, the plates were developed with HRP-conjugated polyclonal antibody to human IgG (DakoCytomation) and o-phenylenediamine substrate. Absorbance was read at $492 \mathrm{~nm}$. The reduced reaction of BPAbs with rhNC16A was expressed as an inhibition rate, which was calculated according to the following formula: inhibition rate $\%=(A 492 b-A 492 f) / A 492 b \times 100$, where $A 492 b$ is the reaction with BPAbs only and A492f is the reaction competed with $\mathrm{Fab}$ at a given concentration.

Inhibition ELISA between phage antibodies (Phabs) and Fabs from the isolated clones was performed to determine whether the Fabs against different epitopes mutually cross-inhibit binding by steric hindrance. Individual Phabs were incubated with rhNC16A on ELISA plates. The reaction was challenged with Fabs from different clones at various concentrations. After washing, the remaining binding of the Phabs to rhNC16A was developed with the HRP-conjugated anti-M13 antibody and o-phenylenediamine substrate.

\section{Inhibition IF}

Inhibition IF was assessed to check the competition of Fabs to the binding of BPAbs by incubating purified BPAbs (10 $\mu \mathrm{g} / \mathrm{ml})$ with 0 to $40 \mu \mathrm{g} / \mathrm{ml}$ Fabs on human skin sections. FITC-conjugated anti-human IgG (DakoCytomation) was the detection reagent. The inhibition IF was also performed by sequential incubation with BPAbs on human skin sections, which was followed by Fabs with a 30-minute interval. The effects of Fab inhibition on the binding of autoantibodies from patients with linear IgA bullous dermatosis and anti-p200 pemphigoid were also observed.

\section{In Vitro Inhibition of BPAb-Induced Complement Activation}

BPAb-induced complement activation in human skin samples and the inhibitory effects of anti-COL17 NC16A Fabs were observed by IF as described by Nelson et al with minor modifications. ${ }^{9}$ Cryosections of normal human skin were incubated with BPAbs $(10 \mu \mathrm{g} / \mathrm{ml})$, anti-COL17 NC16A Fabs (10 to $40 \mu \mathrm{g} / \mathrm{ml}$ ), or BPAbs plus anti-COL17 NC16A Fabs for one hour at $37^{\circ} \mathrm{C}$. Freshly prepared normal human serum was then added as a complete complement source. One hour after incubation, in situ deposition of human $\mathrm{C} 1 \mathrm{q}$ and $\mathrm{C} 3$ at the DEJ was detected with FITC-conjugated mAbs to human $\mathrm{C} 1 \mathrm{q}$ and human C3 (DakoCytomation), respectively.

\section{Effects of Fabs on BP Mouse Model in Vivo}

All mouse procedures were approved by the Institutional Animal Care and Use Committee of Hokkaido University, and the experimental mice were housed in a specific pathogen-free animal facility. The BP model mice were produced by injecting BP autoantibodies, either NC16A affinity purified BPAbs (50 $\mu \mathrm{g} / \mathrm{g}$ body weight) or whole BP-IgG (1 mg/g body weight) prepared from BP patients, into the COL17 humanized neonatal mice, as previously reported. ${ }^{3}$ At 48 hours after the injection, the mice developed human BP-like clinical and histological characteristics with serum autoantibody titers ranging from 1:80 to 1:640 in IIF and a mean BP180 antibody index value of $55.7 \pm 21.1$ by ELISA measurement, which is similar to the autoantibody level usually found in the sera of active BP patients. To observe the effects of the generated 
anti-COL17 NC16A Fabs on the COL17 humanized mice and on the BPAb-induced disease, we divided the neonatal mice into different groups. We first injected Fabs from the three individual clones to test whether the recombinant Fabs themselves are pathogenic in COL17 humanized mice. The Fab doses were 30 to $90 \mu \mathrm{g} / \mathrm{g}$ body weight (30 $\mu \mathrm{g} / \mathrm{g}$ body weight is roughly an equimolar dose compared with $50 \mu \mathrm{g} / \mathrm{g}$ body weight of IgGBPAbs). To sequentially monitor the serum Fab levels after injection, $60 \mu \mathrm{g} / \mathrm{g}$ body weight of Fab-B4 was injected into the neonatal mice and blood samples were collected by sacrificing mice at $6,24,48$, and 72 hours. The Fab concentration was quantified using a sandwich ELISA technique with two mAbs. To capture Fabs in the samples, rabbit anti-c-myc mAb (Santa Cruz Biotechnology) was coated onto ELISA plates (20 $\mu \mathrm{g} / \mathrm{ml}$ in PBS overnight at $4^{\circ} \mathrm{C}$ ). After blocking with $3 \% \mathrm{BSA}$ for one hour at $37^{\circ} \mathrm{C}$, individual serum samples were diluted with $1 \%$ BSA in PBS buffer (1:10) and incubated for 1 hour at $37^{\circ} \mathrm{C}$. Purified Fab was used as a standard at concentrations ranging between $0.1 \mu \mathrm{g} / \mathrm{ml}$ and $50 \mu \mathrm{g} / \mathrm{ml}$. The plate was then incubated with HRP-conjugated mouse antihuman lambda light-chain mAb to detect the reaction. The concentrations of Fabs in the samples were calculated from the standard curve for each plate.

The effects of Fabs on the BP autoantibody-induced mouse model were observed by injecting Fabs either from individual clones or from various combinations of the clones. The injection of antibodies into mice was performed as described previously, with minor modifications. ${ }^{3}$ Briefly, each mouse received a single intraperitoneal injection of different antibodies according to group. At 48 hours after injection, the extent of skin disease was judged, including distinct Nikolsky sign. The animals were then sacrificed, and skin samples were studied by light microscopy and direct immunofluorescence microscopy using FITC conjugated antibodies against human lambda light chain (DakoCytomation), c-myc tag (Santa Cruz Biotechnology), human IgG (Jackson, West Grove, PA), and mouse complement C3 (Cappel, ICN Pharmaceuticals, Inc., Aurora, OH). The quantification of mast cells (MCs) and MC degranulation was performed as described by Nelson et al, and the results were expressed as a percentage of degranulated MCs (number of degranulated MCs per total number of MCs in 5 random fields $\times 100 \%)^{9}$

Blood was collected, and the serum sample was prepared and used for ELISA to determine the titers of circulating BPAbs or Fabs. The level of BPAbs in the serum samples of experimental mice was tested using an antiCOL17 ELISA kit according to the manufacturer's instructions (MBL, Nagoya, Japan). Absorbance was read at $450 \mathrm{~nm}$. The index value was defined by the following formula: index $=(A 450$ of tested serum $-\mathrm{A} 450$ of negative control)/(A450 of positive control - A450 of negative control) $\times 100$. The concentration of the recombinant Fabs in serum samples obtained from the experimental mice was quantified using the sandwich ELISA technique described above.

\section{Statistical Analysis and Ethical Considerations}

Differences in the ELISA inhibition results among various groups were examined for statistical significance using the analysis of variance with Fisher PLSD test. For the analysis of MC degranulation among various groups of Fab treatments, we determined statistical significance using multiple tests including the Student $t$ test and one way analysis of variance. $P$ values less than 0.05 were considered significant.

This study was approved by the Institutional Review Board of Hokkaido University, and fully informed consent from all patients was obtained for use of human material.

\section{Results}

\section{Isolation of Anti-COL17 NC16A Antibodies from Phage Antibody Libraries}

Two individual Fab phage libraries containing $8 \times 10^{7}$ clones and $4 \times 10^{7}$ clones, respectively, were successfully constructed by combining light chain genes and heavy chain genes amplified from antibody repertoires of two BP patients (library 1 from patient 1; library 2 from

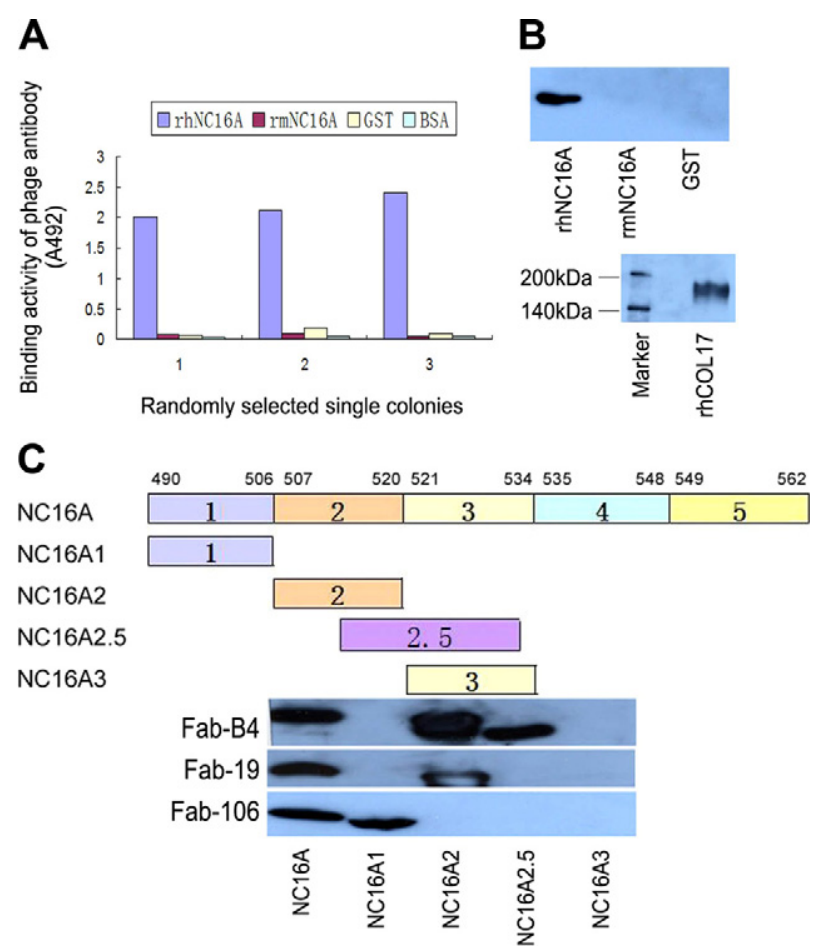

Figure 1. Isolation of specific binders against the NC16A domain of human type XVII collagen from phage antibody library. A: Randomly selected single colonies from the fourth panning round of the libraries show positive reaction with recombinant human NC16A (rhNC16A) but no reaction with recombinant mouse NC16A (rmNC16A), GST, or BSA in ELISA. B: Western blotting of soluble Fabs shows staining of only the rhNC16A domain and full-length human COL17, whereas rmNC16A and GST are negative. The representative results using Fab-B4 are shown. C: Epitope mapping of the generated Fabs with rhNC16A and its subdomains are shown. Fab-B4 binds to rhNC16A, NC16A2, and NC16A2.5 but does not react with NC16A1 and NC16A3, indicating that the binding epitope is located within an overlapping region within subdomain 2 and 2.5 (amino acids 514 to 520). The Fab-106 and Fab-19 epitopes are located in subdomain 1 (NC16A1, amino acids 490 to 506 ) and subdomain 2 (NC16A2, amino acids 507 to 520 ), respectively. 
Table 2. Heavy and Light Chain Genes of Isolated Fabs

\begin{tabular}{|c|c|c|c|c|c|c|c|c|}
\hline \multirow{2}{*}{$\begin{array}{l}\text { Fab } \\
\text { clone }\end{array}$} & \multirow{2}{*}{$\begin{array}{c}\mathrm{VH} \\
\text { family }\end{array}$} & \multicolumn{3}{|c|}{ Amino acid sequences of $\mathrm{VH}$} & \multirow{2}{*}{$\begin{array}{c}V L \\
\text { family }\end{array}$} & \multicolumn{3}{|c|}{ Amino acid sequences of VL } \\
\hline & & CDR1 & CDR2 & CDR3 & & CDR1 & CDR2 & CDR3 \\
\hline B4 & $\mathrm{VH} 1$ & NYAFSW & GIIPMSGEGHKAQKFQG & PSRSNYAGGMDV & $V_{\lambda 1} 1$ & SGSSSNIGRHYVY & TNYRRPS & ASWDDSL \\
\hline B12 & $\mathrm{VH} 3$ & SYSMN & SISSSSSYIYYADSVKG & IDSSSWYEGWFDP & $\mathrm{V} \lambda 1$ & SGSTSNIGSNTVN & SNNQRLS & GTWDDSLN \\
\hline B21 & $\mathrm{VH} 3$ & SYVLS & LLVVMLEADTTQTPEG & GNNWYGQTFDF & $\vee \lambda 1$ & GAAPTSGQVMMYTW & GNSNRPS & QSYDSSLS \\
\hline F32 & $\mathrm{VH} 3$ & SYAMH & VISYDGSNKYYADSVKG & ALRGYSYGT & $V_{\kappa} 1$ & RASQSISSYLN & AASSLQS & QQSYSLF \\
\hline 19 & $\mathrm{VH} 3$ & NYGMH & VISYDGSKKYYADSVKG & GFYYDWGTYYDY & $V \lambda 1$ & TGSSSNIGAGYDVH & ANSNRPS & QSYDSSLT \\
\hline 106 & $\mathrm{VH} 3$ & DSAIH & RVRSKTNNYATDYAVSVKGR & HGESRSWYVGSGYWFDP & $V \lambda 1$ & SGSSSNIGNNYVS & DNNKRPS & GTWDSSLS \\
\hline
\end{tabular}

Six unique antibody clones against the NC16A domain of human COL17 were identified by sequencing the heavy and light chain variable regions. Of these, clones B4, B12, B21, and F32 were isolated from library 1, whereas clones 19 and 106 were isolated from library 2. The deduced amino acids sequences of the complementary determining regions (CDRs) are shown.

patient 2). Phabs were selected by panning against rhNC16A immobilized on immune tubes. ELISA of the Phabs revealed specific positive reactions with rhNC16A in 40 of 96 and 32 of 80 colonies isolated from the two libraries, respectively (Figure 1A). By BstN I fingerprinting and sequencing of variable regions of heavy chain $(\mathrm{VH})$ and light chain $(\mathrm{VL})$ genes, nine unique antibody clones against rhNC16A were identified and were allowed to express the soluble Fab fragments.

\section{Expression and Characterization of Fabs}

Soluble Fab fragments of the nine antibody clones were successfully expressed by removing the gene III fragment of the phagemid vector. Four of the soluble Fabs from library 1 (Fab-B4, Fab-B12, Fab-B21, Fab-F32) and two from library 2 (Fab-19, Fab-106) were highly specific to rhNC16A in ELISA (data not shown) and Western blot analysis (Figure 1B, representative Western blot result). The other three clones, however, could not be detected as soluble fragments, probably because of their low affinity. The $\mathrm{VH}$ and $\mathrm{VL}$ genes of the six positive Fab clones are summarized in Table 2.
By epitope mapping, the binding site of the Fabs with rhNC16A and its subdomains was obtained. All four of the Fabs from library 1 showed the same reactive pattern. They bound to rhNC16A and subdomains 2 and 2.5 but failed to react with subdomains 1 and 3 , indicating that their binding epitope was within the overlapping region (amino acids 514 to 520) of subdomains 2 and 2.5. The two Fabs from library 2 bound to different subdomains: Fab-106 reacted only with subdomain 1 (amino acids 490 to 506) and Fab-19 reacted only with subdomain 2 (amino acids 507 to 520 ). This indicates that they bound to different epitopes on COL17 NC16A. The representative blot results are shown in Figure 1C. These data demonstrate the successful isolation of anti-COL17 NC16A Fabs from patients with BP.

We chose Fabs (Fab-B4, Fab-19, and Fab-106) that had been raised against different epitopes of COL17 NC16A for further experiments. All of the light chains of these three clones are from human lambda light chain family. Largescale production was performed, and a yield of approximately 1 to $3 \mathrm{mg}$ of Fab product was obtained from each 1 $L$ culture after column purification. Figure $2 \mathrm{~A}$ shows the SDS-PAGE identification of the purified Fab in reduced and nonreduced form.
A

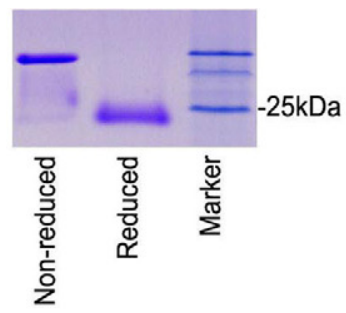

B

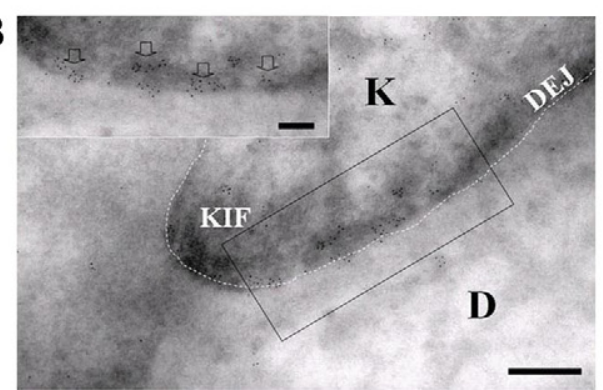

C

C

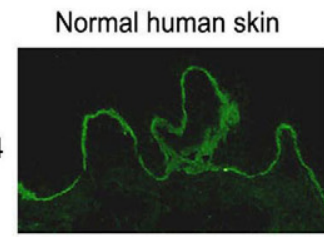

$\mathrm{NaCl}$ split human skin
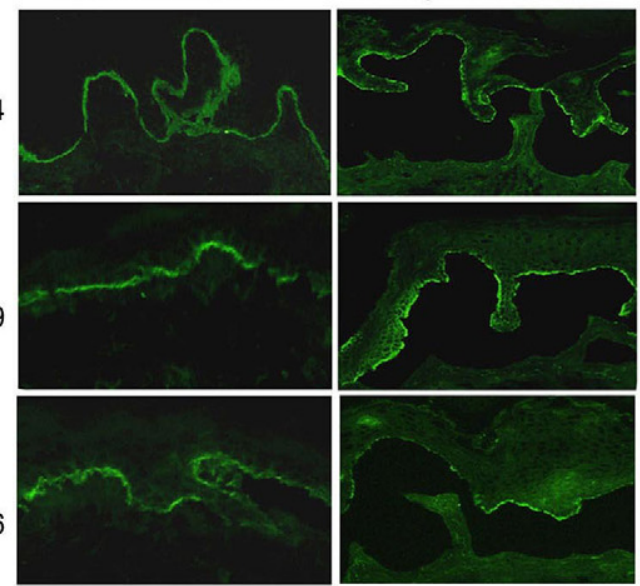

Skin sample from COL17 humanized mouse

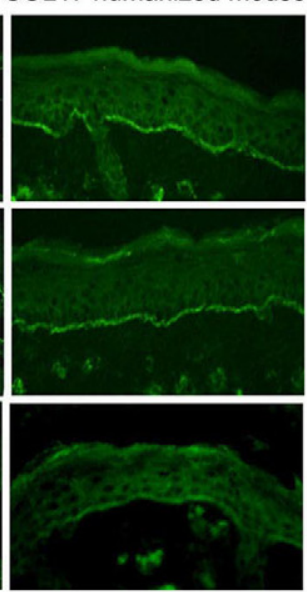

Figure 2. Production and characterization of Fabs. A: A purified soluble Fab in both reduced and nonreduced states is shown by Coomassie blue staining after SDS-PAGE. B: Immunogold labeling of normal human skin by Fabs shows 5-nm immunogold deposits restricted to immediately beneath hemidesmosomes, close to the keratinocyte plasma membrane (arrows, bar $=100 \mathrm{~nm}$ ). The representative results using Fab-B4 are shown. (K: keratinocyte; D: dermis; KIF: keratin intermediate filaments; DEJ: dermal-epidermal junction). C: Immunofluorescence studies on normal human skin and skin sections from COL17 humanized mice show positive staining of the selected Fabs at the DEJ, and positive staining is also noted on the roof of $\mathrm{NaCl}$ split skin samples. 
Table 3. Affinity of Anti-COL17 NC16A Fabs Measured by BIAcore System

\begin{tabular}{cccc}
\hline Fab & $k_{\text {on }}(1 / \mathrm{Ms})$ & $k_{\text {off }}(1 / \mathrm{s})$ & $K_{\mathrm{D}}(\mathrm{M})$ \\
\hline Fab-B4 & $2.83 \times 10^{5}$ & $1.10 \times 10^{-3}$ & $3.89 \times 10^{-9}$ \\
Fab-19 & $1.14 \times 10^{5}$ & $6.26 \times 10^{-3}$ & $5.48 \times 10^{-8}$ \\
Fab-106 & $5.52 \times 10^{5}$ & $8.08 \times 10^{-2}$ & $1.46 \times 10^{-7}$ \\
\hline
\end{tabular}

Kinetic parameters $k_{\text {on }}$ and $k_{\text {off }}$ were measured by BIAcore system, and $K_{\mathrm{D}}$ was calculated as $k_{\text {on }} / k_{\text {off. }}$. From the three Fabs, Fab-B4 has the highest affinity

Immunogold electron microscopy showed that 5-nm immunogold particles were restricted to immediately beneath hemidesmosomes, below the keratinocyte plasma membrane (Figure 2B). Mean measurements of immunogold deposits demonstrated that their epitopes were about 1 to $2 \mathrm{~nm}$ (mean $1.77 \mathrm{~nm} \pm \mathrm{SD}, n>200$ ) beneath the plasma membrane and located between the epitopes of $1 \mathrm{~A} 8 \mathrm{C}$, a cytoplasmic plaque associated COL17 antibody) and 233 (an extracellular COL17 antibody), as described by Nonaka et al. ${ }^{26}$ No difference in distribution of the immunogold deposits was found between the three Fabs.

In the IIF experiments, as we expected, all three Fabs showed linear deposition at the DEJ and positive staining
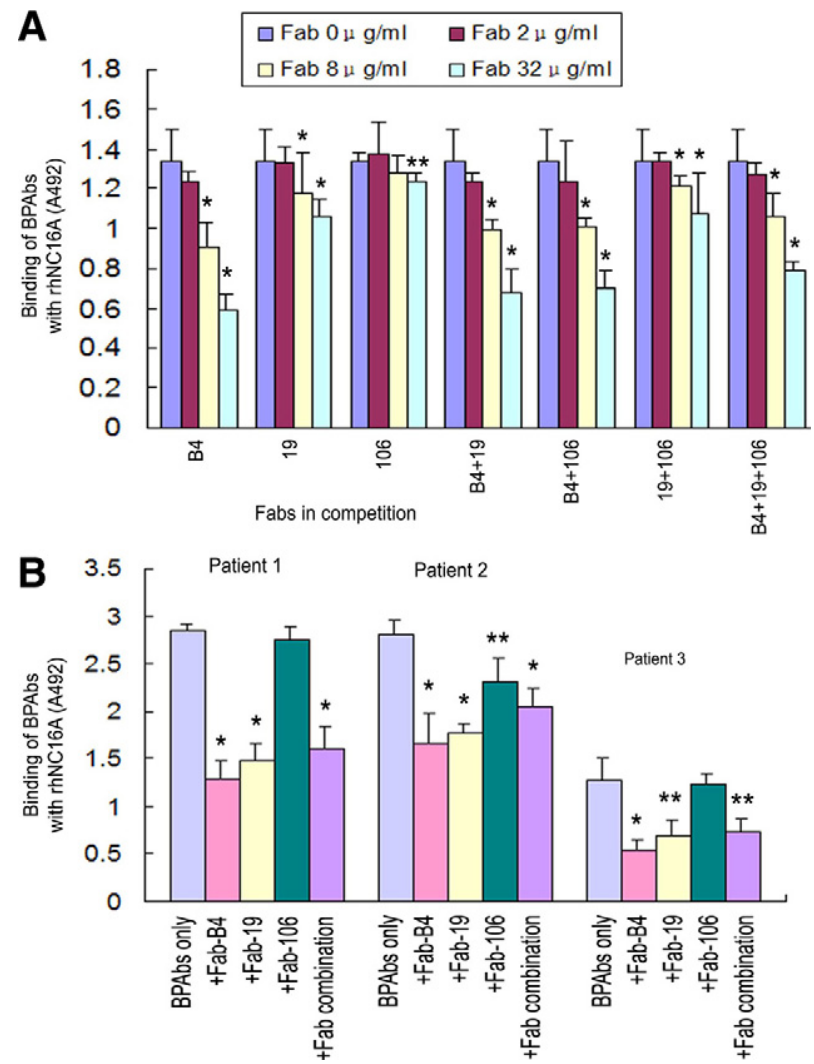

Figure 3. Inhibition ELISA assay. A: The effects of competition with Fab-B4, Fab-19, and various combinations inhibit the binding of autoantibodies (BPAbs, $8 \mu \mathrm{g} / \mathrm{ml}$ ) from pooled sera of patients with bullous pemphigoid (BP) to rhNC16A in a dose-dependent manner (0 to $32 \mu \mathrm{g} / \mathrm{ml})$, whereas Fab-106 inhibits BPAb binding only moderately. ${ }^{*} P<0.01$, ${ }^{* *} P<0.05$, versus the original binding of BPAbs. B: Fabs $(32 \mu \mathrm{g} / \mathrm{ml})$ inhibit the binding of BPAbs from three BP patients. ${ }^{*} P<0.01,{ }^{* *} P<0.05$, versus the original binding of BPAbs. was noted along the roof of the $\mathrm{NaCl}$ split skin samples, consistent with COL17 staining (Figure 2C).

Kinetic analysis using the BIAcore system demonstrated affinity levels of Fab-B4, Fab-19, and Fab-106, as summarized in Table 3. Among these Fabs, Fab-B4 showed the highest affinity value and Fab-106 showed the lowest.

\section{Functional Analysis of Fabs in Vitro}

To determine whether the Fabs generated against COL17 NC16A were able to function in competitive binding to inhibit the emergence of an autoantibody-mediated $\mathrm{BP}$ phenotype, we initially performed a series of in vitro experiments to evaluate their ability to block BP autoantibody binding to COL17. Figure $3 \mathrm{~A}$ shows that the rhNC16A binding activities of BPAbs, which were affinity purified using recombinant COL17 NC16A peptide from the pooled sera from $20 \mathrm{BP}$ patients, were reduced markedly and significantly by Fab-B4 and Fab-19, but only marginally by Fab-106, in a dose-dependent manner ( 0 to $32 \mu \mathrm{g} / \mathrm{ml}$ ). Fab-B4 and 19 suppressed the binding of BPAbs most efficiently at a concentration of $32 \mu \mathrm{g} / \mathrm{ml}$, with the highest inhibition rates of $52.4 \%$ and $50.8 \%$, respectively. Combinations of two or three Fabs failed to increase this inhibition efficacy. When tested with BPAbs isolated from individual BP patients, Fabs showed similar competitive effects (Figure 3B).

IIF studies show competitive blocking of Fabs with BPAbs on skin sections. Figure 4 shows positive IgG
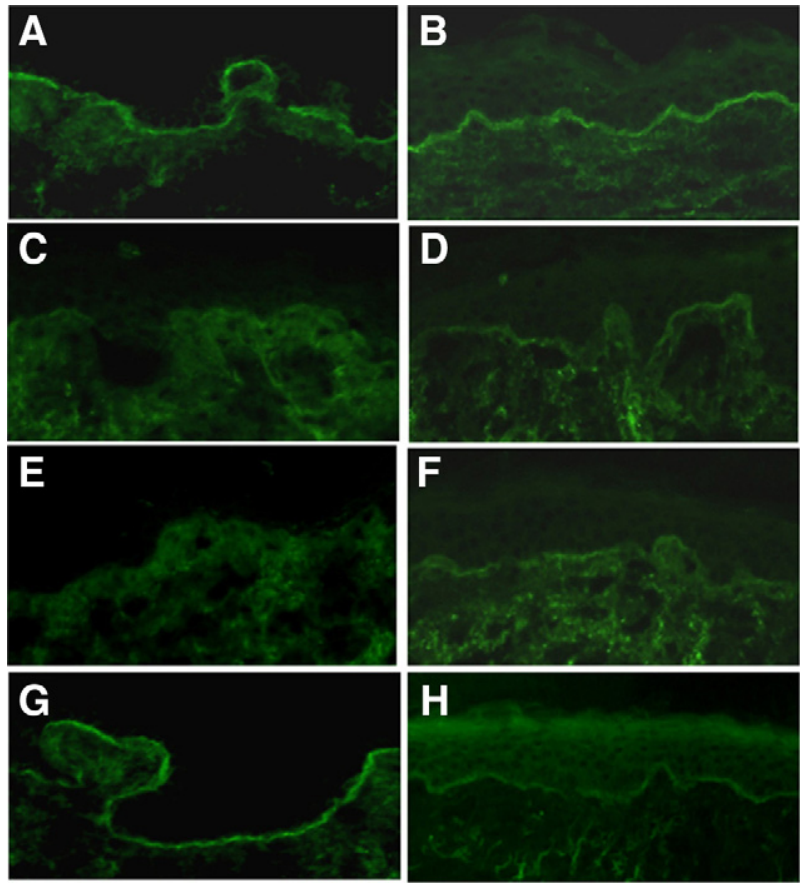

Figure 4. Inhibition immunofluorescence. A and B: Positive IgG staining of the NC16A affinity purified BPAbs $(10 \mu \mathrm{g} / \mathrm{ml})$ at the DEJ in human skin. C and E: IgG BPAbs staining is blocked by coincubation with either Fab-B4 or Fab-19 at a concentration of $20 \mu \mathrm{g} / \mathrm{ml}$. G: Fab-106 $(20 \mu \mathrm{g} / \mathrm{ml})$ fails to significantly inhibit the binding of BPAbs. When BPAbs are allowed to bind to skin sections first and Fabs are added 30 minutes later, the IF staining of BPAb binding is also markedly reduced by Fab-B4 (D) or Fab-19 (F) but not by Fab-106 (H). 

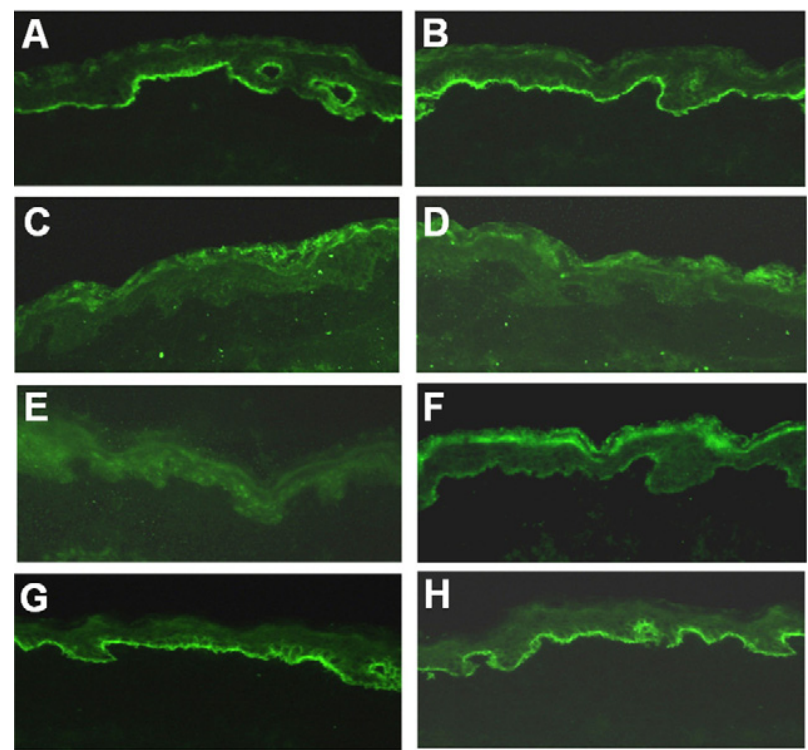

Figure 5. BPAb-induced complement activation and the inhibitory effects of Fabs. NC16A affinity purified BPAbs $(10 \mu \mathrm{g} / \mathrm{ml})$ induced activation of human $\mathrm{C} 1 \mathrm{q}$ and $\mathrm{C} 3$ is shown at the DEJ in cryosections of human skin (A and $\mathbf{B}$ ). When Fabs are coadministered with BPAbs at the same concentration, Fab-B4 completely blocks C1q and C3 activation (C and D), whereas Fab-19 effectively blocks the activation of $\mathrm{C} 1 \mathrm{q}(\mathbf{E})$ and markedly reduces the activation of C3 (F). Fab-106 shows no inhibition of either C1q or C3 activation ( $\mathbf{G}$ and $\mathbf{H}$ ).

BPAbs staining $(10 \mu \mathrm{g} / \mathrm{ml})$ at the DEJ in human skin (Figure 4A), which was blocked by coincubation with either Fab-B4 or Fab-19 at a concentration of $20 \mu \mathrm{g} / \mathrm{ml}$ (Figure 4, C and E). Fab-106 failed to significantly inhibit the binding of BPAbs (Figure 4G). When BPAbs were allowed to bind to skin sections first and Fabs were added 30 minutes later, the IF staining of BPAb binding was also markedly reduced although not completely inhibited (Figure 4, B, D, F, and H). Competitive IF using Fabs and individual patient BPAbs showed that Fab-B4 and Fab-19 were able to block the binding of autoantibodies from three individual BP patients, whereas none of the Fabs inhibited the binding of IgA autoantibodies from patients with linear IgA bullous dermatosis or IgG autoantibodies from patients with anti-p200 pemphigoid (data not shown).

In vitro inhibition of complement activation by recombinant Fabs was studied by immunofluorescence. In situ deposition of BPAb-activated C1q (Figure 5A) and C3 (Figure 5B) was found at the DEJ in human skin. Complement deposition was reduced or completely blocked by Fab-B4 (Figure 5, C and D) or Fab-19 (Figure 5, E and F), whereas it was unchanged by Fab-106 treatment (Figure 5, G and H). Fabs against COL17 NC16A did not activate complement at concentrations up to $100 \mu \mathrm{g} / \mathrm{ml}$.

We also tested the effect of competition between recombinant anti-COL17 NC16A Fabs. Using an inhibition ELISA, Fabs from the three clones inhibited the binding of Phabs from their own clone as we might have expected. Interestingly, Fab-B4 and Fab-19 cross-inhibited each other while Fab-106 failed to inhibit the other two (Figure 6, A-C). These data indicate that Fab-B4 and Fab-19 specifically recognize distinct but close or overlapping
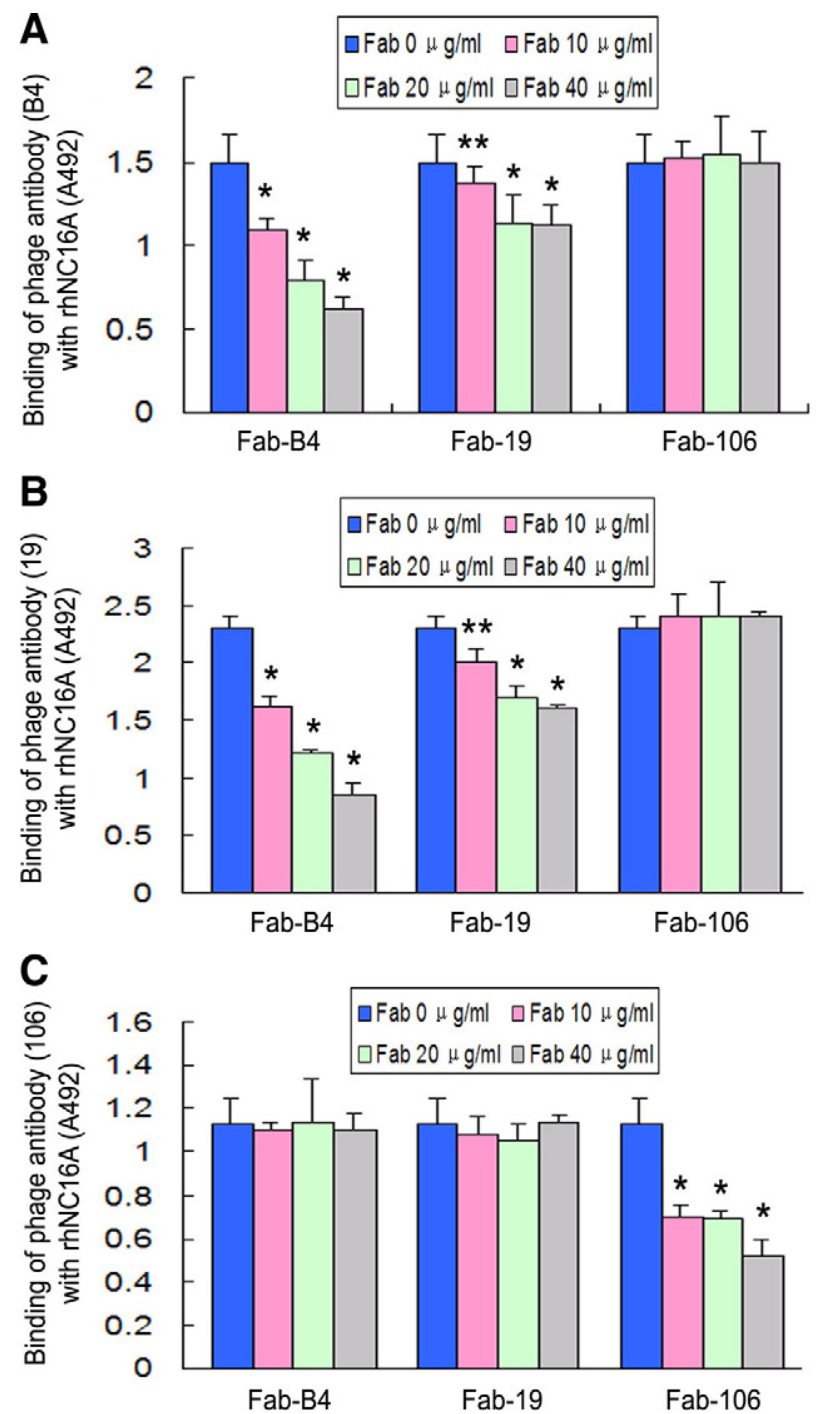

Figure 6. Inhibition ELISA for the three recombinant antibody clones using Fabs and phage antibodies (Phabs). Fab-B4 and Fab-19 inhibited the binding of both Phab-B4 and Phab-19 (A and B), whereas Fab-106 inhibited Phab-106 only (C). This indicates that the antibody clones B4 and 19 are mutually cross-inhibiting. ${ }^{*} P<0.01,{ }^{* *} P<0.05$, versus the original binding of respective Phabs.

epitopes and are able to block the binding of BP antibodies in nearby epitopes, most likely by direct steric hindrance.

\section{In Vivo Blockade of Autoantibody-Induced BP}

\section{Disease}

We first proved that recombinant Fabs were not pathogenic to COL17 humanized mice after injection with 30 to $90 \mu \mathrm{g} / \mathrm{g}$ body weight of Fab-B4, -19, or -106 into neonatal mice. Neither clinical signs, including erythema and Nikolsky sign, nor any histopathological manifestations of BP were found in the treated mice. Direct immunofluorescence studies demonstrated clear deposition of the recombinant Fab fragments at the DEJ. Subsequent deposition of mouse C3 was not detected (Figure $7 \mathrm{~A}$ ). 
A
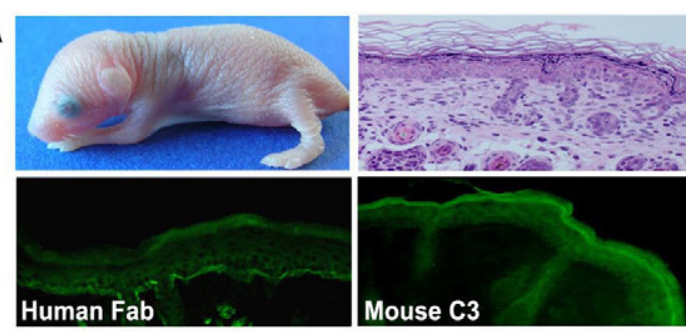

Effect of Fab-mAb on COL17 humanized mouse

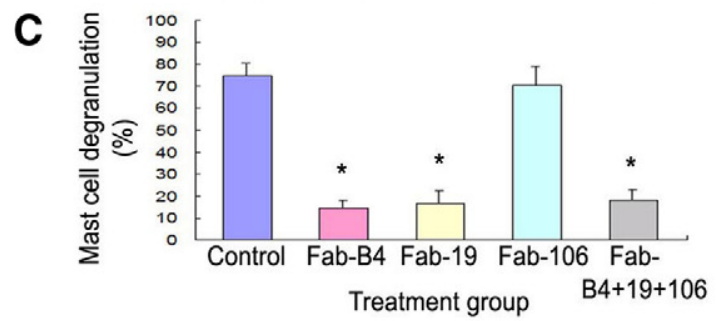

D

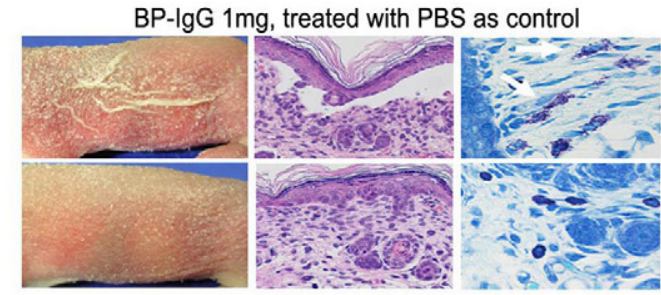

BP-IgG 1mg, followed by Fab-B4 $50 \mu \mathrm{g}$

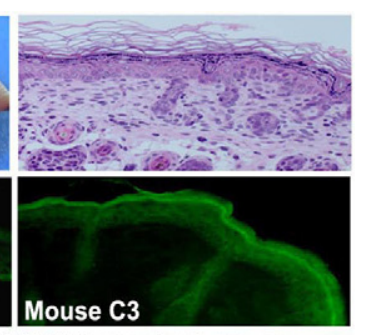

B
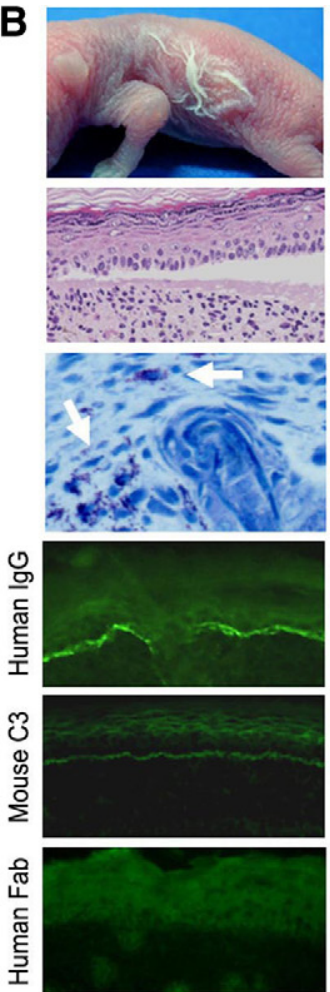

BPAbs
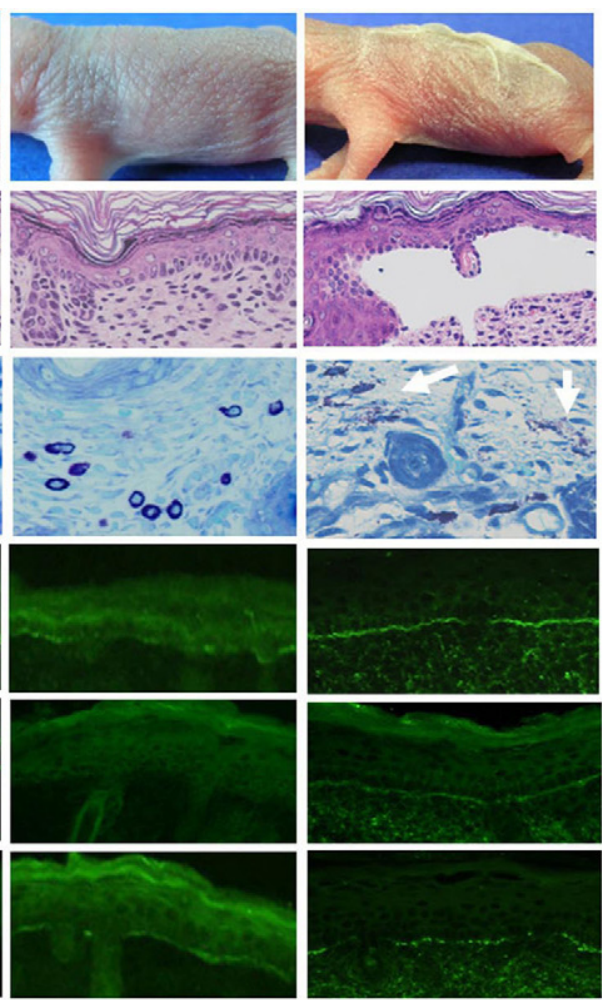

BPAbs+Fab-B4

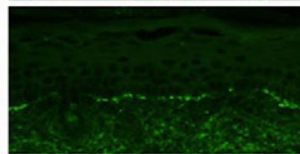

BPAbs+Fab-106

Figure 7. Therapeutic effects of Fabs on BP model mice. A: Results of the injection of Fabs into neonatal COL17 humanized mice show that this treatment alone does not cause BP disease or other detectable adverse effects. Histological examination (right, upper panel) supports this result. Indirect immunofluorescence show DEJ staining for the recombinant Fabs (left of lower panel) but no staining for mouse C3 (right, lower panel). B: Mice injected with NC16A affinity purified BPAbs develop the clinical and histological skin detachment associated with MC degranulation (white arrows) and the deposition of human IgG and mouse $\mathrm{C} 3$ at the DEJ. In contrast, mice injected with BPAbs and Fab-B4 fail to show these clinical and histological characteristics, and the intensity of IgG deposition at the DEJ is markedly reduced. The staining of mouse C3 is absent, whereas recombinant Fab fragment staining is weak but detectable. Fab-106 fails to show any beneficial therapeutic effect in the animal model. C: Percentage of dermal MC degranulation is assessed in BP model mice and in those treated with $30 \mu \mathrm{g} / \mathrm{g}$ body weight of Fabs. It is significantly reduced in the mice treated with Fab-B4, Fab-19, and the Fab combination. ${ }^{*} P<0.01$ versus control group (BP model mice treated with PBS). D: BP model mice were produced by injection of BP-IgG (total IgG fraction prepared from BP patients) and were treated with Fab-B4 24 hours later. BP-like clinical and histological characteristics fail to develop in most (four of five) of Fab-B4 treated mice (lower panel).

For further in vivo experiments, we again used the humanized BP mouse model and induced disease by injecting BP autoantibodies, either BPAbs (50 $\mu \mathrm{g} / \mathrm{g}$ body weight) or whole BP-lgG (1 mg/g body weight), into neonatal COL17 humanized mice. We sequentially analyzed the serum concentration of BP autoantibodies in the injected mice. Their autoantibody titers ranged from 1:80 to $1: 640$ in IIF, and the mean BP180 antibody index value reached the highest level of $72.8 \pm 21.7$ at 6 hours and then gradually decreased to $68.5 \pm 9.1$ at 12 hours, $64.1 \pm 14.9$ at 24 hours, $55.7 \pm 21.1$ at 48 hours, $33.4 \pm$ 4.8 at 72 hours, and $33.7 \pm 2.6$ at 96 hours after injection. After i.p. injection of BPAbs or whole BP-lgG, the mice were i.p. treated either immediately or 24 hours later with Fabs from each of the three individual clones. The results were evaluated 48 hours later (Table 4). As shown in Figure 7B, the BP model mice untreated with Fabs demonstrated a BP-like clinical phenotype with extensive erythema and Nikolsky sign together with histological characteristics, including dermal-epidermal separation and the infiltration of inflammatory cells. These clinical and histological signs failed to develop in any of the Fab-B4 groups, including the immediately treated and the 24 hour-treated mice. Treatment with Fab-19 demonstrated a similar effect. Fab-106, however, failed to show any therapeutic efficacy. Histologically, no subepidermal blister formation was found in skin samples from mice treated with Fab-B4 or -19 at a dosage of $30 \mu \mathrm{g} / \mathrm{g}$ body weight or higher, whereas distinctive BP-like blister formation was observed in skin sections from the majority of the Fab-106 treated mice (4/5 mice with the $30 \mu \mathrm{g} / \mathrm{g}$ body weight treatment and $4 / 4$ mice with the $60 \mu \mathrm{g} / \mathrm{g}$ body weight treatment) or control mice. Fab combination therapy at the same total dose showed a similar result as treatment with Fab-B4 or -19 alone. Direct immunofluorescence studies revealed that deposition of human IgG and mouse $\mathrm{C} 3$ at the DEJ was significantly reduced in the Fab-B4 or -19 treated groups compared with that of controls. Extensive MC degranulation took place in the dermis of the BP model mice. In contrast, the percentage of degranulated MCs was significantly decreased in the Fab-B4- and -19-treated mice (Figure 7C). Figure 7D shows the BP disease phenotype induced by injection of whole BP-IgG (upper panel) and the therapeutic results of Fab (lower panel). Fab-B4 treatment inhibited the BPIgG-induced phenotypic changes in all immediately treated mice, as well as the majority of the mice $(4 / 5)$ treated 24 hours after the initial BP-IgG injection. To- 
Table 4. Effects of Anti-COL17 NC16A Fabs on BP Autoantibody-Induced Mouse Model

\begin{tabular}{|c|c|c|c|}
\hline $\begin{array}{c}\text { Abs used for reproducing } \\
\text { mouse model }\end{array}$ & Treatment & $\begin{array}{l}\text { Total dose of Fab } \\
(\mu \mathrm{g} / \mathrm{g} \text { body weight) }\end{array}$ & $\begin{array}{c}\text { Skin } \\
\text { detachment }\end{array}$ \\
\hline \multirow{14}{*}{$\begin{array}{l}\text { COL17 NC16A- affinity purified BP } \\
\text { autoantibodies (BPAbs) }\end{array}$} & \multirow[t]{4}{*}{ Fab-B4 } & 15 & $2 / 8$ \\
\hline & & 30 & $0 / 10$ \\
\hline & & $\underline{30 \text { (24 hours later) }}$ & $\underline{0 / 4}$ \\
\hline & & 60 & $\overline{0 / 9}$ \\
\hline & \multirow[t]{3}{*}{ Fab-19 } & 15 & $2 / 6$ \\
\hline & & 30 & $0 / 6$ \\
\hline & & 60 & $0 / 6$ \\
\hline & \multirow[t]{3}{*}{ Fab-106 } & 15 & $5 / 5$ \\
\hline & & 30 & $4 / 5$ \\
\hline & & 60 & $4 / 4$ \\
\hline & \multirow[t]{3}{*}{ Fab combination (Fab-B4 + $19+106)$} & 15 & $2 / 5$ \\
\hline & & 30 & $0 / 6$ \\
\hline & & 60 & $0 / 5$ \\
\hline & Control & PBS & $14 / 14$ \\
\hline \multirow[t]{3}{*}{ Whole IgG fractions (BP-IgG) } & Fab-B4 & 30 & $0 / 4$ \\
\hline & \multirow{2}{*}{ Control } & 30 (24 hours later) & $1 / 5$ \\
\hline & & PBS & $\overline{4 / 4}$ \\
\hline
\end{tabular}

Neonatal COL17 humanized BP model mice were injected with either COL17 NC16A-affinity purified BP autoantibodies (BPAbs: 50 mg/g body weight) or whole IgG fractions (BP-lgG: $1 \mathrm{mg} / \mathrm{g}$ body weight) from patients with bullous pemphigoid. The mice were then treated immediately or 24 hours later (underlined) with Fabs by intraperitoneal injection. For Fab combination therapy, the total dose comprised one third of each Fab clone.

gether with the in vitro inhibition IF data, these results demonstrate that Fabs can at least partially displace the bound BP autoantibodies within the DEJ and block BP disease after initial binding not only by NC16A-purified BPAbs but also by combined whole IgG sera fractions from BP patients. When the mice were sacrificed at 48 hours, serum samples were collected to detect BP antibody index values and the recombinant Fab level. The BP180 antibody index value in the control mice sera was $170.3 \pm 26.2$, and no significant increase or decrease was found in any of the treatment groups $(P>0.05)$. The concentrations of Fabs ranged from $1.34 \pm 0.11 \mu \mathrm{g} / \mathrm{ml}$ to $10.22 \pm 0.35 \mu \mathrm{g} / \mathrm{ml}$, increasing with increasing injected dose; however, there was no significant difference between the mice injected with Fabs only and the BP model mice treated with Fabs at the same given doses $(P>$ 0.05). No adverse reactions to the Fab treatments were observed.

\section{Discussion}

In the present study, we successfully generated Fabs against the human COL17 NC16A domain from phage display antibody repertoires derived from two BP patients. These Fabs specifically recognize different epitopes located in NC16A subdomains and competitively inhibit the binding of human BP autoantibodies to COL17. Our novel anti-COL17 NC16A Fabs were observed to inhibit the changes in the BP mouse model that would otherwise have been induced by the injection of human BP autoantibodies into recently engineered COL17-humanized mice.

$\mathrm{BP}$ is the most common autoimmune blistering skin disease. The mortality rate of $\mathrm{BP}$ in various reports ranges from $20 \%$ to $40 \%$, and death is more commonly related to other underlying illness, debilitation associated with severe BP condition, or adverse effects of treatment. ${ }^{27}$ Autoantibodies against two hemidesmosomal antigens,
BPAG1 (BP230) and BPAG2 (COL17 or BP180), have been identified in BP. COL17 autoantibodies are generally thought to play a critical role in the initial pathogenesis of the disease. COL17, a type II transmembrane protein, is the main pathogenic target for BP autoantibodies and the NC16A domain was subsequently confirmed as the main binding epitope. ${ }^{15,28-30}$ COL17 autoantibodies have been widely studied and have been shown to induce activation of complement via a classical pathway that is essential for disease development. This allowed us to devise a new approach toward treating $\mathrm{BP}$ and a therapeutic strategy for such treatment. Using molecular and recombinant protein techniques, we identified three Fabs, Fab-B4, Fab-19, and Fab-106, which recognize multiple epitopes within the COL17 NC16A domain with different affinity levels. Of these, Fab-B4 and Fab-19 recognized distinct epitopes located within subdomain 2 of NC16A, whereas Fab-106 recognized an epitope in subdomain 1. Interestingly, Fab-B4 and Fab-19 inhibited each other in competition ELISA assays using reciprocal Phabs and soluble Fabs, indicating that they bind specifically to their corresponding epitopes and may involve mutual steric hindrance. Fab-106 showed no inhibitory effects on the other two Fabs, confirming its unique binding domain.

Fab-B4 and Fab-19, but not Fab-106, showed therapeutic potential for BP in both in vitro and in vivo studies. Both fragments competitively inhibit the binding of $\mathrm{BP}$ autoantibodies to the main COL17 NC16A epitope, and both of them block BPAb-mediated activation of complement $\mathrm{C} 1 \mathrm{q}$ and $\mathrm{C} 3$. In our BP animal model using the COL17 humanized mouse, marked inhibition of the BP phenotype, including deposition of BPAbs and complement, degranulation of MCs, and subcutaneous blister formation, was observed in Fab-B4- or Fab-19-treated mice. It appears from these data that complete blocking of BP autoantibodies by Fabs is not required to significantly inhibit disease severity. We know this because 
successful treatment of the BP model mouse was achieved even with partial blocking of BP autoantibody binding. This is consistent with the clinical observation that BP symptoms can markedly improve as the autoantibody levels gradually decrease, although autoantibodies are still detectable. ${ }^{31,32}$ This suggests that there may be a threshold for BP autoantibodies to initiate and maintain complement activation and complement-mediated tissue injury. Partially reducing autoantibody deposition using blocking Fabs might be beneficial in alleviating BP. Furthermore, our Fab was demonstrated to be able to displace the bound BP autoantibodies both in vitro and in vivo. These results strongly support our approach of using inhibitory anti-COL17 NC16A Fabs for BP therapy. When comparing the in vitro and in vivo activities of all these Fabs together, the Fab-B4 clone appears to be most efficient. We assessed the effects of combining these three Fabs, but we failed to improve on the results. The inhibitory effect of Fab clones in combination was not as good as that of the Fab-B4 clone alone, even at equivalent dosages. The administration of Fabs themselves or in combination failed to elicit any adverse pathological manifestation in the COL17 humanized mice.

It has been shown that the majority of anti-COL 17 autoantibody pathogenic epitopes are mainly distributed in subdomains 1 to 3 of NC16A. ${ }^{33}$ Fairley et $\mathrm{al}^{23}$ further defined subdomain 2 as the major epitope recognized by both IgG and IgE autoantibodies from BP patients. We tested the reactivity of BP autoantibodies and found that the binding amount and affinity against subdomain 2 was higher than the binding amount and affinity for subdomains 1 and 3 (data not shown). Taken together with the fact that anti-subdomain 2 Fabs show excellent therapeutic effects in BP model mice, even after the disease was induced not only by the COL17 NC16A affinity purified BPAbs, but also by whole BP-lgG fractions, we speculate that the BP autoantibodies that recognize the non-NC16A epitopes may be less pathogenic than those that recognize the NC16A epitopes. The BP autoantibodies that recognize $\mathrm{NC16A}$, especially the subdomain 2 region, are the main pathogenic autoantibodies in BP. Previously reported in vitro studies using cryosections ${ }^{34}$ and in vivo animal models ${ }^{10}$ both suggested that anti NC16A autoantibodies are major pathogenic antibodies in $\mathrm{BP}$, which further supports our speculation.

Thus far, the complement system has been an attractive therapeutic target for a wide range of autoimmune and inflammatory diseases. ${ }^{2,13}$ There are different strategies of inhibiting complement activation. In a previous study, we tried using a recombinant peptide containing BP pathogenic epitopes as a decoy to block both the binding of BP autoantibodies and the activation of complement. ${ }^{3}$ Although it proved effective, a potential pitfall exists. The peptide may act as an antigen and trigger a more severe immune response, and hence, result in further production of pathogenic autoantibodies. Fab therapy eliminates such concerns. Furthermore, it is highly disease-specific and does not involve systemic immune suppression; therefore, it may be used either as an individual therapy or in combination with other currently available treatments to promote efficacy and reduce adverse reactions. Our success in generating these Fabs with therapeutic potential makes it possible to create not only a more specific therapy for BP but also further potential strategies for the treatment of many other antibody-initiated complement-mediated autoimmune disorders.

\section{Acknowledgments}

We thank Ms. Noriko Ikeda, Ms. Maki Goto, Ms. Megumi Sato, and Ms. Akari Nagasaki for their technical assistance.

\section{References}

1. Jacobson DL, Gange SJ, Rose NR, Graham NM: Epidemiology and estimated population burden of selected autoimmune diseases in the United States. Clin Immunol Immunopathol 1997, 84:223-243

2. Holers VM: The complement system as a therapeutic target in autoimmunity. Clin Immunol 2003,107:140-151

3. Nishie W, Sawamura D, Goto M, Ito K, Shibaki A, McMillan JR, Saka K, Nakamura H, Olasz E, Yancey KB, Akiyama M, Shimizu H: Humanization of autoantigen. Nat Med 2007, 13:378-383

4. Liu Z, Giudice GJ, Swartz SJ, Fairley JA, Till GO, Troy JL, Diaz LA: The role of complement in experimental bullous pemphigoid. J Clin Invest 1995, 95:1539-1544

5. Mewar D, Wilson AG: Autoantibodies in rheumatoid arthritis: a review. Biomed Pharmacother 2006, 60:648-655

6. D'Cruz DP, Khamashta MA, Hughes GRV: Systemic lupus erythematosus. Lancet 2007, 369:587-596

7. Girardi G, Berman J, Redecha P, Spruce L, Thurman JM, Kraus D, Hollmann TJ, Casali P, Caroll MC, Wetsel RA, Lambris JD, Holers VM Salmon JE: Complement C5a receptors and neutrophils mediate fetal injury in the antiphospholipid syndrome. J Clin Invest 2003, 112:1644-1654

8. Zhao M, Trimbeger ME, Li N, Diaz LA, Shapiro SD, Liu Z: Role of FcRs in animal model of autoimmune bullous pemphigoid. J Immunol 2006, 177:3398-3405

9. Nelson KC, Zhao M, Schroeder PR, Li N, Wetse RA, Diaz LA, Liu Z Role of different pathways of the complement cascade in experimental bullous pemphigoid. J Clin Invest 2006, 116:2892-2900

10. Liu Z, Diaz LA, Troy JL, Taylor AF, Emery DJ, Fairley JA, Giudice GJ A passive transfer model of the organ-specific autoimmune disease, bullous pemphigoid, using antibodies generated against the hemidesmosomal antigen BP180. J Clin Invest 1993, 92:2480-2488

11. Liu Z: Bullous Pemphigoid: Using animal models to study the immunopathology. J Investig Dermatol Symp Proc 2004, 9:41-46

12. Yancey KB: The pathophysiology of autoimmune blistering diseases. $\mathrm{J}$ Clin Invest 2005, 115:825-828

13. Girardi G, Redecha P, Salmon JE: Heparin prevents antiphospholipid antibody-induced fetal loss by inhibiting complement activation. Nat Med 2004, 10:1222-1226

14. Atkinson JP: Complement system on the attack in autoimmunity. J Clin Invest 2003, 112:1639-1641

15. Giudice GJ, Emery DJ, Zelickson BD, Anhalt GJ, Liu Z, Diaz LA: Bullous pemphigoid and herpes gestationis autoantibodies recognize a common non-collagenous site on the BP180 ectodomain. J Immunol 1993, 151:5742-5750

16. Wang Z, Wang Y, Li Z, Li J, Dong Z: Humanization of a mouse monoclonal antibody neutralizing TNF- $\alpha$ by guided selection. $\mathrm{J}$ Immunol Methods 2000, 241:171-184

17. Barbas III CF, Kang AS, Lerner RA, Benkovic SJ: Assembly of combinatorial antibody libraries on phage surfaces: The gene III site. Proc Natl Acad Sci USA 1991, 88:7978-7982

18. Little M, Breitling F, Dubel S, Fuchs P, Braunagel M: Human antibody libraries in Escherichia coli. J Biotechnol 1995, 41:187-195

19. Clackson T, Hoogenboom HR, Griffiths AD, Winter G: Making antibody fragments using phage display libraries. Nature 1991, 352: 624-628

20. Wang G, Liu YF, Li CY, Lu N, Gao TW, Hua B, Wang Y: Cloning and 
characterization of antikeratin human antibodies using a semisynthetic phage antibody library. Arch Dermatol Res 2004, 296:270-277

21. Marks JD, Hoogenboom HR, Bonnert TP, McCafferty J, Griffiths AD, Winter G: Bypassing immunization of human antibodies from V-gene libraries displayed on phage. J Mol Biol 1991, 222:581-597

22. Hoogenboom HR: Selecting and screening recombinant antibody libraries. Nat Biotechnol 2005, 23:1105-1116

23. Fairley JA, Fu CL, Giudice GJ: Mapping the binding sites of antiBP180 immunoglobulin E autoantibodies in bullous pemphigoid. J Invest Dermatol 2005, 125:467-472

24. Shimizu H, Masunaga T, Ishiko A, Hashimoto T, Garrod DR, Shida H, Nishikawa T: Demonstration of desmosomal antigens by electron microscopy using cryofixed and cryosubstituted skin with silver-enhanced gold probe. J Histochem Cytochem 1994, 42:687-692

25. McMillan JR, Akiyama M, Nakamura $H$, Shimizu $H$ : Colocalization of multiple laminin isoforms predominantly beneath hemidesmosomes in the upper lamina densa of the epidermal basement membrane. $\mathrm{J}$ Histochem Cytochem 2006, 54:109-111

26. Nonaka S, Ishiko A, Masunaga T, Akiyama M, Owaribe K, Shimizu H, Nishikawa T: The extracellular domain of BPAG2 has a loop structure in the carboxy terminal flexible tail in vivo. J Invest Dermatol 2000, 115:889-892

27. Khumalo N, Kirtschig G, Middleton P, Hollis S, Wojnarowska F, Murrell D: Interventions for bullous pemphigoid. Cochrane Database Syst Rev 2005 (3):CD002292

28. Schumann H, Baetge J, Tasanen K, Wojnarowska F, Schäcke H, Zillikens D, Bruckner-Tuderman L: The shed ectodomain of collagen
$\mathrm{XVII/BP} 180$ is targeted by autoantibodies in different blistering skin diseases. Am J Pathol 2000, 156:685-695

29. Ishiko A, Shimizu H, Kikuchi A, Ebihara T, Hashimoto T, Nishikawa T: Human autoantibodies against the $230-\mathrm{kD}$ bullous pemphigoid antigen (BPAG1) bind only to the intracellular domain of the hemidesmosome, whereas those against the $180-\mathrm{kD}$ bullous pemphigoid antigen (BPAG2) bind along the plasma membrane of the hemidesmosome in normal human and swine skin. J Clin Invest 1993, 91:1608-1615

30. Stanley JR: Autoantibodies against adhesion molecules and structures in blistering skin diseases. J Exp Med 1995, 181:1-4

31. Kobayashi M, Amagai M, Kuroda-Kinoshita K, Hashimoto T, Shirakata Y, Hashimoto K, Nishikawa T: BP180 ELISA using bacterial recombinant NC16a protein as a diagnostic and monitoring tool for bullous pemphigoid. J Dermatol Sci 2002, 30:224-232

32. Tsuji-Abe Y, Akiyama M, Yamanaka Y, Kikuchi T, Sato-Matsumura $\mathrm{KC}$, Shimizu $\mathrm{H}$ : Correlation of clinical severity and ELISA indices for the NC16A domain of BP180 measured using BP180 ELISA kit in bullous pemphigoid. J Dermatol Sci 2005, 37:145-149

33. Zillikens D, Rose PA, Balding SD, Liu Z, Olague-Marchan M, Diaz LA, Giudice GJ: Tight clustering of extracellular BP180 epitopes recognized by bullous pemphigoid autoantibodies. J Invest Dermatol 1997, 109:573-579

34. Sitaru C, Schmidt E, Petermann S, Munteanu LS, Brocker EB, ZIllikens $D$ : Autoantibodies to bullous pemphigoid antigen 180 induce dermalepidermal separation in cryosections of human skin. J Invest Dermatol 2002, 118:664-671 